\title{
VORTEX PATCH PROBLEM FOR STRATIFIED EULER EQUATIONS*
}

\author{
TAOUFIK HMIDI ${ }^{\dagger}$ AND MOHAMED ZERGUINE ${ }^{\ddagger}$
}

\begin{abstract}
We study in this paper the vortex patch problem for the stratified Euler equations in space dimension two. We generalize Chemin's result [J.Y. Chemin, Oxford University Press, 1998.] concerning the global persistence of the Hölderian regularity of the vortex patches. Roughly speaking, we prove that if the initial density is smooth and the initial vorticity takes the form $\omega_{0}=\mathbf{1}_{\Omega}$, with $\Omega$ a $C^{1+\varepsilon}$-bounded domain, then the velocity of the stratified Euler equations remains Lipschitz globally in time and the vorticity is split into two parts $\omega(t)=\mathbf{1}_{\Omega_{t}}+\widetilde{\rho}(t)$, where $\Omega_{t}$ denotes the image of $\Omega$ by the flow and has the same regularity of the domain $\Omega$. The function $\widetilde{\rho}$ is a smooth function.
\end{abstract}

Key words. Stratified system, vortex patches, para-differential calculus, time decay.

AMS subject classifications. 35Q35, 35B65, 76D03.

\section{Introduction}

This paper deals with the vortex patch problem for the two-dimensional stratified Euler equations described by the following system:

$$
\left\{\begin{array}{l}
\partial_{t} v+v \cdot \nabla v+\nabla \pi=\rho e_{2}, \quad(t, x) \in \mathbb{R}_{+} \times \mathbb{R}^{2}, \\
\partial_{t} \rho+v \cdot \nabla \rho-\Delta \rho=0 \\
\operatorname{div} v=0, \\
v_{\mid t=0}=v_{0}, \quad \rho_{\mid t=0}=\rho_{0} .
\end{array}\right.
$$

Here, the unknowns are the velocity $v=\left(v^{1}, v^{2}\right)$ which is a time-dependent vector field on $\mathbb{R}^{2}$, the pressure $\pi$ is a scalar function, and $\rho$ denotes the temperature or the density. The vector $e_{2}$ is given by $(0,1)$. This system is a simplified model often used to describe the atmosphere and ocean dynamics. It arises from the density-dependent fluid equations by using the so-called Boussinesq approximation which neglects the variation in density everywhere except in the buoyancy term. In dimension two the vorticity $\omega$ of the velocity $v$ is a scalar and takes the form $\omega=\partial_{1} v^{2}-\partial_{2} v^{1}$. Applying the differential operator curl to the first equation of (1.1) we shall have formally an equivalent form of this system written in the vorticity-density formulation.

$$
\left\{\begin{array}{l}
\partial_{t} \omega+v \cdot \nabla \omega=\partial_{1} \rho, \quad(t, x) \in \mathbb{R}_{+} \times \mathbb{R}^{2} \\
\partial_{t} \rho+v \cdot \nabla \rho-\Delta \rho=0 \\
v=\nabla^{\perp} \Delta^{-1} \omega \\
\omega_{\mid t=0}=\omega_{0}, \rho_{\mid t=0}=\rho_{0} .
\end{array}\right.
$$

This system is a generalization of Euler equations obtained with a constant density and it has attracted a lot of attention in the last decade. Many results dealing with the global well-posedness problem were recently obtained by numerous authors and we will restrict our discussion here to some of them. In [3], Chae proved the global well-posedness in the Sobolev framework, namely $\left(v_{0}, \rho_{0}\right) \in H^{s} \times H^{s}, s>2$. This

*Received: August 13, 2012; accepted (in revised form): November 16, 2013. Communicated by Shi Jin.

†IRMAR, Université de Rennes 1, Campus de Beaulieu, 35042 Rennes cedex, France (thmidi@ univ-rennes1.fr).

${ }^{\ddagger}$ Université Hadj Lakhdar Batna, Faculté des Sciences, Département de Mathématiques, LEDPA, 05000 Batna, Algérie (mohamed_zerguine@yahoo.fr). 
result was later improved in [13] by the first author and Keraani for critical Besov regularities. In [7], Danchin and Paicu proved that we can go beyond the strong solutions and establish the global existence and uniqueness for weak initial data in the weak sense. More recently, the authors have established a global well-posedness result for (1.1) with fractional dissipation, namely when the usual Laplacian $-\Delta$ is replaced by $(-\Delta)^{\frac{\alpha}{2}}$, with $\left.\left.\alpha \in\right] 1,2\right]$. The critical case $\alpha=1$ was solved later in [14] by using the special structure of the equations. For more information about this subject and other connected models we refer the reader to the papers $[8,15,17,18,19]$ and the references therein.

One of the main goals of this paper is to study the vortex patch problem for the stratified Euler equations. As we shall see soon this is a special class of Yudovich solutions where the velocity develops a good behavior and remains in the Lipschitz class. Before giving more precise details about this problem we will give first an overview of the vortex patch problem for planar incompressible ideal fluid. According to the theory of Yudovich [4], the $2 d$ Euler system is globally well-posed if the initial vorticity $\omega_{0} \in L^{1} \cap L^{\infty}$, and because the vorticity is transported by the flow it must be constant along particle trajectories. As a consequence when the initial vorticity has a vortex patch structure, that is the characteristic function of a bounded domain $\Omega$, the vorticity will preserve the same structure through time. This means that $\omega(t)=\mathbf{1}_{\psi(t, \Omega)}$, where the flow map $\psi$ is defined by the differential equation

$$
\left\{\begin{array}{l}
\partial_{t} \psi(t, x)=v(t, \psi(t, x)) \\
\psi(0, x)=x
\end{array}\right.
$$

Now what about the regularity of the domain $\psi(t, \Omega)$ ? From Yudovich's work we know that the flow is not in general Lipschitz and may exhibit a breaking regularity $\psi(t) \in C^{e^{-\alpha t}}$. Unfortunately this cannot give useful information about the regularity of the patch. In [4], Chemin proved that if the initial boundary belongs to the class $C^{1+\varepsilon}$, with $0<\varepsilon<1$, the patch will preserve this regularity for any positive time. In order to get this result Chemin proved a logarithmic estimate which relates the Lipschitz norm of the velocity to the co-normal regularity of the vorticity. For more details about this subject we refer the reader to the references $[1,6,9,10,11]$. A special case of Chemin's result reads as follows.

THEOREM 1.1. Let $v_{0}$ be a divergence-free vector field such that its vorticity $\omega_{0}=\mathbf{1}_{\Omega}$ with $\Omega$ a $C^{1+\varepsilon}$-bounded domain with $\left.\varepsilon \in\right] 0,1[$. Then the Euler system has a unique global solution such that

$$
\|\nabla v(t)\|_{L^{\infty}} \leq C_{0} e^{C_{0} t} .
$$

Moreover, the domain $\psi(t, \Omega)$ is of class $C^{1+\varepsilon}$ for every $t \geq 0$.

Let us now come back to the stratified Euler system described by (1.1). Our goal is to analyze the vortex patch problem for this model and to get precise information about the time evolution of the initial patch. As we have already seen, the vorticity in $2 d$ Euler system remains a patch for every time and especially it does not lose its regularity when it is better than $C^{1}$. Even though this structure is instantaneously altered for the stratified Euler system due to the effects of the gravitational force, it will be of interest to investigate whether or not the flow image of the initial domain $\Omega_{t} \triangleq \Psi(t, \Omega)$ preserves its Hölderian regularity. We shall give here a positive answer to this problem and show in addition that the vorticity can be decomposed into a singular part which is a vortex patch term and a regular part connected to the 
smoothing effects of density. We shall also give some asymptotic behavior for large time which is needed to measure the difference between the vorticity and its singular part.

Our main result reads as follows.

THEOREM 1.2. Let $\Omega$ be a $C^{1+\varepsilon}$-bounded domain, with $0<\varepsilon<1, \omega_{0}=\mathbf{1}_{\Omega}$, and $\rho_{0} \in L^{1} \cap L^{\infty}$. Then the system (1.1) admits a unique global solution $(v, \rho)$ such that

$$
(v, \rho) \in L_{\text {loc }}^{\infty}\left(\mathbb{R}_{+} ; L i p\right) \times L^{\infty}\left(\mathbb{R}_{+} ; L^{1} \cap L^{\infty}\right), \quad\|\nabla v(t)\|_{L^{\infty}} \leq C_{0} e^{C_{0} t \log ^{2}(1+t)} .
$$

If $\psi$ denotes the flow associated to the velocity $v$, then for any $t \geq 0$ the domain $\Omega_{t}=$ $\psi(t, \Omega)$ belongs to the class $C^{1+\varepsilon}$. Moreover we have the following decomposition:

$$
\omega(t, x)=\mathbf{1}_{\Omega_{t}}+\widetilde{\rho}(t, x)
$$

with $\widetilde{\rho} \in L_{l o c}^{\infty}\left(\mathbb{R}_{+} ; C^{\eta}\right), \forall \eta<1$ and

$$
\left\|\omega(t)-\mathbf{1}_{\Omega_{t}}\right\|_{L^{2}} \leq C_{0} \log (1+t), \quad\|\widetilde{\rho}(t)\|_{C^{\eta}} \leq C_{0} e^{\exp C_{0} t \log ^{2}(1+t)} .
$$

Here Lip denotes the Lipschitz class and $C_{0}$ a constant depending on the initial data.

Now a few remarks are in order.

\section{REMARKS 1.3.}

1. The $L^{\infty}$-estimate of the vorticity that we are able to obtain has a logarithmic growth for large time which is not optimal compared to the incompressible Euler system. More precisely, we obtain

$$
\|\omega(t)\|_{L^{\infty}} \leq C_{0} \log ^{2}(1+t) .
$$

This explains the additional logarithmic factor in the growth of the gradient of the velocity.

2. It is interesting to know whether or not the logarithmic growth for the difference between the vorticity and its singular part is optimal.

The proof of Theorem 1.2 follows the formalism developed by Chemin in [4] for the Euler system. It is shown, and this is the main tool of Chemin's work, that only the co-normal regularity of the vorticity $\partial_{X} \omega$ contributes to the Lipschitz norm of the velocity through a logarithmic estimate. To encode the co-normal regularity we construct a suitable time-dependent vector field $\left(X_{t}\right)$ which should be tangent for any time to the image of the initial boundary by the flow map $\psi(t)$. Therefore $\left(X_{t}\right)$ should satisfy the transport equation,

$$
\partial_{t} X+v \cdot \nabla X=X \cdot \nabla v .
$$

One of the main feature of these vector fields is the commutation property with the transport operator $\partial_{t}+v \cdot \nabla$, which leads in turn to

$$
\left(\partial_{t}+v \cdot \nabla\right) \partial_{X} \omega=\partial_{X} \partial_{1} \rho .
$$

For the incompressible Euler equation the last term $\partial_{X} \partial_{1} \rho$ disappears and therefore the co-normal derivative of the vorticity is simply transported by the flow. In our context we should deal carefully with this additional term where we lose formally two derivatives on the density. The maximal smoothing effects for the transport-diffusion 
model governing the density equations could lead to suitable estimates, allowing us to prove the global estimate for the Lipschitz norm. However we found it more convenient to diagonalize the system to reduce the loss to just one derivative for the density and not two. This is inspired by the recent work [14]. More precisely, we introduce the coupled function $\Gamma \triangleq \omega-\partial_{1} \Delta^{-1} \rho$ which satisfies the transport equation

$$
\partial_{t} \Gamma+v \cdot \nabla \Gamma=\left[\partial_{1} \Delta^{-1}, v \cdot \nabla\right] \rho \triangleq F .
$$

Consequently we get

$$
\left(\partial_{t}+v \cdot \nabla\right) \partial_{X} \Gamma=\partial_{X} F .
$$

Formally we can easily see that $F$ is of order zero with respect to $\rho$ according to the smoothing effect of the singular operator $\partial_{1} \Delta^{-1}$. Thus instead of manipulating $\partial_{X} \partial_{1} \rho$ in the equation (1.3) we need to understand the commutator which is a nonlinear term but exhibits a good behavior in $\rho$. The advantage of this approach concerns its flexibility to be applied for fractional stratified Euler equations where the Laplacian can be replaced by $(-\Delta)^{\frac{\alpha}{2}}$ with $\alpha \in[1,2]$.

Another task which is raised in the second part of Theorem 1.2 concerns the description of the vorticity, which can be decomposed in a singular part of patch type and a smooth one related to the density. This is done by using the smoothing effects combined with the asymptotic behavior of the solutions for large time. For this latter problem we need to perform suitable decay estimates for transport-diffusion model which are almost optimal compared to the heat equation due to a logarithmic loss; see for example Proposition 4.2.

Our paper is structured as follows. In Section 2 we recall some basic results on the para-differential calculus and state some useful lemmas. Section 3 is devoted to the establishment of some commutator estimates. In Section 4 we prove some asymptotic behavior for Yudovich solutions. The last section is concerned with the proof of our main result.

\section{Basic tools}

2.1. Notations. Throughout this work we will use the following notations.

- We denote by $C$ a positive constant which may be different in each occurrence but does not depend on the initial data. We shall sometimes alternatively use the notation $X \lesssim Y$ for an inequality of type $X \leq C Y$ with $C$ independent of $X$ and $Y$. The notation $C_{0}$ means a constant depending on the involved norms of the initial data.

- For any tempered distribution $u$ both $\widehat{u}$ and $\mathcal{F} u$ denote the Fourier transform of $u$.

- For every $p \in[1, \infty],\|\cdot\|_{L^{p}}$ denotes the usual norm of the Lebesgue space $L^{p}$.

- The mixed space-time Lebesgue space $L^{p}\left([0, T], L^{r}\left(\mathbb{R}^{d}\right)\right)$ is equipped with the norm $\|\cdot\|_{L_{T}^{p} L^{r}}$ (with the obvious generalization to $\|\cdot\|_{L_{T}^{p} \mathcal{X}}$ for any Banach space $\mathcal{X}$ ).

- For any pair of operators $P$ and $Q$, the commutator $[P, Q]$ is given by $P Q-Q P$.

2.2. Functional spaces. We shall in what follows recall the definition of Hölder spaces $C^{n+\alpha}\left(\mathbb{R}^{d}\right)$. For $\left.\alpha \in\right] 0,1\left[\right.$, we denote by $C^{\alpha}\left(\mathbb{R}^{d}\right)$ the set of continuous functions $u$ such that

$$
\|u\|_{C^{\alpha}}=\|u\|_{L^{\infty}}+\sup _{x \neq y} \frac{|u(x)-u(y)|}{|x-y|^{\alpha}}<\infty .
$$


We will also make use of the space $C^{1+\alpha}\left(\mathbb{R}^{d}\right)$ which is the set of continuously differentiable functions $u$ such that

$$
\|u\|_{C^{1+\alpha}}=\|u\|_{L^{\infty}}+\|\nabla u\|_{C^{\alpha}}<\infty .
$$

By the same way we can define generally the spaces $C^{n+\alpha}$, with $n \in \mathbb{N}$ and $\left.\alpha \in\right] 0,1[$.

Now we introduce the so-called Littlewood-Paley decomposition and the corresponding cut-off operators which are the basic ingredients for the para-differential calculus and the characterization of Besov spaces. There exist two radial positive functions $\chi \in \mathcal{D}\left(\mathbb{R}^{d}\right)$ and $\varphi \in \mathcal{D}\left(\mathbb{R}^{d} \backslash\{0\}\right)$ such that

i) $\chi(\xi)+\sum_{q \geq 0} \varphi\left(2^{-q} \xi\right)=1 ; \quad \forall q \geq 1, \operatorname{supp} \chi \cap \operatorname{supp} \varphi\left(2^{-q}\right)=\varnothing$,

ii) $\operatorname{supp} \varphi\left(2^{-j} \cdot\right) \cap \operatorname{supp} \varphi\left(2^{-k} \cdot\right)=\varnothing$, if $|j-k| \geq 2$.

For every $v \in \mathcal{S}^{\prime}\left(\mathbb{R}^{d}\right)$ we set the cut-off operators,

$$
\Delta_{-1} v=\chi(\mathrm{D}) v ; \forall q \in \mathbb{N}, \Delta_{q} v=\varphi\left(2^{-q} \mathrm{D}\right) v \quad \text { and } S_{q}=\sum_{-1 \leq p \leq q-1} \Delta_{p} .
$$

From [2], we split the product $u v$ of two distributions into three parts,

$$
u v=T_{u} v+T_{v} u+R(u, v)
$$

with

$$
T_{u} v=\sum_{q} S_{q-1} u \Delta_{q} v, \quad R(u, v)=\sum_{q} \Delta_{q} u \tilde{\Delta}_{q} v \quad \text { and } \quad \tilde{\Delta}_{q}=\sum_{j=-1}^{1} \Delta_{q+j} .
$$

Let us now introduce the Besov spaces.

Definition 2.1. For $(p, r) \in[1,+\infty]^{2}$ and $s \in \mathbb{R}$ we define the inhomogeneous Besov space $B_{p, r}^{s}$ as the set of tempered distributions $u$ such that

$$
\|u\|_{B_{p, r}^{s}} \triangleq\left(2^{q s}\left\|\Delta_{q} u\right\|_{L^{p}}\right)_{\ell^{r}}<\infty .
$$

Notice that the usual Sobolev space $H^{s}$ coincides with $B_{2,2}^{s}$ for any $s \in \mathbb{R}$ and that the Hölder space $C^{n+\alpha}$ coincides with the Besov space $B_{\infty, \infty}^{n+\alpha}$

We shall also use some mixed space-time spaces.

Definition 2.2. Letting $T>0$ and $\rho \geq 1$, we denote by $L_{T}^{\rho} B_{p, r}^{s}$ the space of distributions $u$ such that

$$
\|u\|_{L_{T}^{\rho} B_{p, r}^{s}} \triangleq\left\|\left(2^{q s}\left\|\Delta_{q} u\right\|_{L^{p}}\right)_{\ell^{r}}\right\|_{L_{T}^{\rho}}<\infty
$$

We say that $u$ belongs to the space $\widetilde{L}_{T}^{\rho} B_{p, r}^{s}$ if

$$
\|u\|_{\widetilde{L}_{T}^{\rho} B_{p, r}^{s}} \triangleq\left(2^{q s}\left\|\Delta_{q} u\right\|_{L_{T}^{\rho} L^{p}}\right)_{\ell^{r}}<\infty .
$$

REMARK 2.3. By an immediate application of the Minkowski inequality, we have the following embeddings. If $\varepsilon>0$, then

$$
L_{T}^{\rho} B_{p, r}^{s} \hookrightarrow \widetilde{L}_{T}^{\rho} B_{p, r}^{s} \hookrightarrow L_{T}^{\rho} B_{p, r}^{s-\varepsilon}, \quad \text { if } r \geq \rho,
$$




$$
L_{T}^{\rho} B_{p, r}^{s+\varepsilon} \hookrightarrow \widetilde{L}_{T}^{\rho} B_{p, r}^{s} \hookrightarrow L_{T}^{\rho} B_{p, r}^{s}, \quad \text { if } \quad \rho \geq r .
$$

We shall make continuous use of Bernstein inequalities; for the proof see for instance [4].

Lemma 2.4. There exists a constant $C$ such that for $q, k \in \mathbb{N}, 1 \leq a \leq b$, and for $f \in L^{a}\left(\mathbb{R}^{d}\right)$,

$$
\begin{aligned}
\sup _{\substack{|\alpha|=k \\
C^{\prime}}}\left\|\partial^{\alpha} S_{q} f\right\|_{L^{b}} \leq C^{k} 2^{q\left(k+d\left(\frac{1}{a}-\frac{1}{b}\right)\right)}\left\|S_{q} f\right\|_{L^{a}} \\
C^{-k} 2^{q k}\left\|\Delta_{q} f\right\|_{L^{a}} \leq \sup _{|\alpha|=k}\left\|\partial^{\alpha} \Delta_{q} f\right\|_{L^{a}} \leq C^{k} 2^{q k}\left\|\Delta_{q} f\right\|_{L^{a}} .
\end{aligned}
$$

To end this paragraph we state the following result whose proof can be found in $[4,11]$.

Proposition 2.5. Let $s \in]-1,1\left[,(p, r) \in[1, \infty]^{2}\right.$, and $v$ be a divergence-free vector field belonging to $L_{\mathrm{loc}}^{1}\left(\mathbb{R}_{+} ; \operatorname{Lip}\left(\mathbb{R}^{d}\right)\right)$. Let a be a solution of

$$
\partial_{t} a+v \cdot \nabla a-\nu \Delta a=f .
$$

Then the following estimate holds true:

$$
\|a(t)\|_{B_{p, r}^{s}} \leq C e^{C V(t)}\left(\|a(0)\|_{B_{p, r}^{s}}+\int_{0}^{t} e^{-C V(\tau)}\|f(\tau)\|_{B_{p, r}^{s}} d \tau\right),
$$

with $V(t)=\int_{0}^{t}\|\nabla v(\tau)\|_{L^{\infty}} d \tau$ and $C$ a constant depending only on $s$ and $d$.

\section{Commutator estimates}

We start this section with the following technical lemma whose proof can be found in [14].

Lemma 3.1. Let $(a, b) \in[1, \infty]^{2}$ such that $a \geq b^{\prime}$ with $\frac{1}{b}+\frac{1}{b^{\prime}}=1$. Given $f, g$, and $h$ three functions such that $\nabla f \in L^{a}, g \in L^{b}$, and $x h \in L^{b^{\prime}}$, then

$$
\|h \star(f g)-f(h \star g)\|_{L^{a}} \lesssim\|x h\|_{L^{b^{\prime}}}\|\nabla f\|_{L^{a}}\|g\|_{L^{b}} .
$$

Now, we introduce the operator $\mathcal{L} \triangleq \partial_{1} \Delta^{-1}$, which is of convolution type, and our aim is to estimate in a suitable space the commutator between this singular operator and the convective one $v \cdot \nabla$. We point out that the operator $\mathcal{L}$ will appear in Section 5 when we deal with the evolution of a vortex patch.

Lemma 3.2. Let $\varepsilon \in] 0,1[, \rho$ be a smooth function and $v$ be a smooth divergence-free vector field on $\mathbb{R}^{2}$ with vorticity $\omega$. Assume that $v \in L^{2}, \omega \in L^{2} \cap L^{\infty}$, and $\rho \in L^{2} \cap L^{p}$, with $p>\frac{2}{1-\varepsilon}$. Then the following statement holds true:

$$
\|[\mathcal{L}, v \cdot \nabla] \rho\|_{C^{\varepsilon}} \lesssim\|v\|_{L^{2}}\|\rho\|_{L^{2}}+\|\omega\|_{L^{2} \cap L^{\infty}}\|\rho\|_{L^{p}}
$$

Proof. According to Bony's decomposition,

$$
[\mathcal{L}, v \cdot \nabla] \rho=\sum_{q \in \mathbb{N}}\left[\mathcal{L}, S_{q-1} v \cdot \nabla\right] \Delta_{q} \rho+\sum_{q \in \mathbb{N}}\left[\mathcal{L}, \Delta_{q} v \cdot \nabla\right] S_{q-1} \rho+\sum_{q \geq-1}\left[\mathcal{L}, \Delta_{q} v \cdot \nabla\right] \tilde{\Delta}_{q} \rho
$$




$$
\begin{aligned}
& \triangleq \sum_{q \in \mathbb{N}} \Pi_{1}^{q}+\sum_{q \in \mathbb{N}} \Pi_{2}^{q}+\sum_{q \geq-1} \Pi_{3}^{q} \\
& \triangleq \Pi_{1}+\Pi_{2}+\Pi_{3} .
\end{aligned}
$$

To estimate the first term $\Pi_{1}^{q}$ we use its convolution structure,

$$
\Pi_{1}^{q}=h_{q} \star\left(S_{q-1} v \Delta_{q} \nabla \rho\right)-S_{q-1} v\left(h_{q} \star \nabla \Delta_{q} \rho\right),
$$

where $\widehat{h}_{q}(\xi)=\frac{\xi_{1}}{|\xi|^{2}} \psi\left(2^{-q} \xi\right)$ and $\psi$ is a smooth function supported in an annulus with center zero. Therefore $h_{q}(x)=2^{q} h\left(2^{q} x\right)$ with $h \in \mathcal{S}$. In view of the Lemma 3.1 we get

$$
\left\|\Pi_{1}^{q}\right\|_{L^{\infty}} \lesssim\left\|x h_{q}\right\|_{L^{p^{\prime}}}\left\|\nabla S_{q-1} v\right\|_{L^{\infty}}\left\|\Delta_{q} \nabla \rho\right\|_{L^{p}} .
$$

Using the fact $\left\|x h_{q}\right\|_{L^{p^{\prime}}}=2^{-2 \frac{q}{p^{\prime}}}\|x h\|_{L^{p^{\prime}}}$ combined with the Bernstein inequality we obtain

$$
\begin{aligned}
\left\|\Pi_{1}^{q}\right\|_{L^{\infty}} & \lesssim 2^{q\left(-1+\frac{2}{p}\right)}\left\|\Delta_{q} \rho\right\|_{L^{p}} \sum_{-1 \leq j \leq q-2}\left\|\nabla \Delta_{j} v\right\|_{L^{\infty}} \\
& \lesssim 2^{q\left(-1+\frac{2}{p}\right)}\|\rho\|_{L^{p}}\left(\left\|\nabla \Delta_{-1} v\right\|_{L^{2}}+(q-1)\|\omega\|_{L^{\infty}}\right) \\
& \lesssim 2^{q\left(-1+\frac{2}{p}\right)}\|\rho\|_{L^{p}}\left(\|\omega\|_{L^{2}}+(q-1)\|\omega\|_{L^{\infty}}\right) .
\end{aligned}
$$

In the last line we have used that $\Delta_{j}$ maps $L^{p}$ into itself uniformly in $j$ and $p$ together with the following equivalence:

$$
\left\|\Delta_{q} v\right\|_{L^{\infty}} \approx 2^{-q}\left\|\Delta_{q} \omega\right\|_{L^{\infty}} \quad \forall q \in \mathbb{N} .
$$

Because

$$
\Delta_{j} \sum_{q \in \mathbb{N}} \Pi_{1}^{q}=\sum_{|j-q| \leq 4} \Pi_{1}^{q}
$$

and with the assumption $p>\frac{2}{1-\varepsilon}$,

$$
\begin{aligned}
\left\|\Pi_{1}\right\|_{C^{\varepsilon}} & \lesssim \sup _{j} \sum_{|j-q| \leq 4} 2^{q \varepsilon}\left\|\Pi_{1}^{q}\right\|_{L^{\infty}} \\
& \lesssim\left(\|\omega\|_{L^{2}}+\|\omega\|_{L^{\infty}}\right)\|\rho\|_{L^{p}} .
\end{aligned}
$$

Concerning the second term $\Pi_{2}^{q}$, we do not need to use the structure of the commutator. We write

$$
\begin{aligned}
\Pi_{2}^{q} & =\mathcal{L}\left(\Delta_{q} v \cdot \nabla S_{q-1} \rho\right)-\Delta_{q} v \cdot \nabla \mathcal{L}\left(S_{q-1} \rho\right) \\
& \triangleq \Lambda_{1}^{q}+\Lambda_{2}^{q} .
\end{aligned}
$$

For $\Lambda_{1}^{q}$ we use that $\mathcal{L}$ is a homogeneous operator of order -1 and the Fourier transform of $\Delta_{q} v \cdot S_{q-1} \nabla \rho$ is supported in an annulus of size $2^{q}$. Then we get, by making use of Bernstein inequality,

$$
\begin{aligned}
\left\|\Lambda_{1}^{q}\right\|_{L^{\infty}} & \lesssim 2^{-q}\left\|\Delta_{q} v\right\|_{L^{\infty}}\left\|S_{q-1} \nabla \rho\right\|_{L^{\infty}} \\
& \lesssim 2^{q\left(-1+\frac{2}{p}\right)}\|\omega\|_{L^{\infty}}\|\rho\|_{L^{p}} .
\end{aligned}
$$


Because the Riesz transform $\nabla \mathcal{L}$ continuously maps $L^{p}$ into itself with $1<p<\infty$, this yields

$$
\begin{aligned}
\left\|\Lambda_{2}^{q}\right\|_{L^{\infty}} & \lesssim 2^{-q}\left\|\Delta_{q} \nabla v\right\|_{\infty}\left\|S_{q-1} \nabla \mathcal{L} \rho\right\|_{L^{\infty}} \\
& \lesssim 2^{q\left(-1+\frac{2}{p}\right)}\|\omega\|_{L^{\infty}}\|\rho\|_{L^{p}} .
\end{aligned}
$$

Putting together (3.2) and (3.3) we get

$$
\left\|\Pi_{2}^{q}\right\|_{L^{\infty}} \lesssim 2^{q\left(-1+\frac{2}{p}\right)}\|\omega\|_{L^{\infty}}\|\rho\|_{L^{p}}
$$

Now we can conclude, as with the first term $\Pi_{1}$,

$$
\left\|\Pi_{2}\right\|_{C^{\varepsilon}} \lesssim\|\omega\|_{L^{\infty}}\|\rho\|_{L^{p}}
$$

Let us now move to the third term $\Pi_{3}$. According to the definition of the remainder term one writes

$$
\begin{aligned}
\Pi_{3} & =\sum_{q \in \mathbb{N}}\left[\mathcal{L}, \Delta_{q} v \cdot \nabla\right] \tilde{\Delta}_{q} \rho+\left[\mathcal{L}, \Delta_{-1} v \cdot \nabla\right] \tilde{\Delta}_{-1} \rho \\
& =\left\{\sum_{q \in \mathbb{N}} \mathcal{L} \operatorname{div}\left(\Delta_{q} v \tilde{\Delta}_{q} \rho\right)-\sum_{q \in \mathbb{N}} \Delta_{q} v \cdot \nabla \mathcal{L}\left(\tilde{\Delta}_{q} \rho\right)\right\}+\left[\mathcal{L}, \Delta_{-1} v \cdot \nabla\right] \tilde{\Delta}_{-1} \rho \\
& =\Pi_{3}^{1}+\Pi_{3}^{2} .
\end{aligned}
$$

By Bernstein's inequality we obtain successively

$$
\begin{aligned}
\left\|\Delta_{j} \mathcal{L} \operatorname{div}\left(\Delta_{q} v \tilde{\Delta}_{q} \rho\right)\right\|_{L^{\infty}} & \lesssim 2^{j \frac{2}{p}}\left\|\Delta_{j} \mathcal{L} \operatorname{div}\left(\Delta_{q} v \tilde{\Delta}_{q} \rho\right)\right\|_{L^{p}} \\
& \lesssim 2^{j \frac{2}{p}}\left\|\Delta_{q} v\right\|_{L^{\infty}}\left\|\tilde{\Delta}_{q} \rho\right\|_{L^{p}} \\
& \lesssim 2^{-q+j \frac{2}{p}}\|\omega\|_{L^{\infty}}\|\rho\|_{L^{p}}
\end{aligned}
$$

and

$$
\begin{aligned}
\left\|\Delta_{j}\left(\Delta_{q} v \cdot \nabla \mathcal{L}\left(\tilde{\Delta}_{q} \rho\right)\right)\right\|_{L^{\infty}} & \lesssim 2^{j \frac{2}{p}}\left\|\Delta_{q} v\right\|_{L^{\infty}}\left\|\nabla \mathcal{L}\left(\tilde{\Delta}_{q} \rho\right)\right\|_{L^{p}} \\
& \lesssim 2^{-q+j \frac{2}{p}}\|\omega\|_{L^{\infty}}\left\|\tilde{\Delta}_{q} \rho\right\|_{L^{p}} \\
& \lesssim 2^{-q+j \frac{2}{p}}\|\omega\|_{L^{\infty}}\|\rho\|_{L^{p}}
\end{aligned}
$$

We multiply both sides of (3.5) and (3.6) by $2^{\varepsilon j}$ and take the supremum over $j \geq-1$ to find

$$
\begin{aligned}
\left\|\Pi_{3}^{1}\right\|_{C^{\varepsilon}} & =\sup _{j \geq-1} 2^{\varepsilon j}\left\|\Delta_{j} \Pi_{3}^{1}\right\|_{L^{\infty}} \\
& \lesssim\|\omega\|_{L^{\infty}}\|\rho\|_{L^{p}} \sup _{j \geq-1} \sum_{q \geq j-4} 2^{-q+j\left(\varepsilon+\frac{2}{p}\right)} \\
& \lesssim\|\omega\|_{L^{\infty}}\|\rho\|_{L^{p}} \sup _{j \geq-1} 2^{j\left(-1+\varepsilon+\frac{2}{p}\right)} \\
& \lesssim\|\omega\|_{L^{\infty}}\|\rho\|_{L^{p}}
\end{aligned}
$$

To estimate the last term $\Pi_{3}^{2}$ it would be better to transform it under the form

$$
\Pi_{3}^{2}=\operatorname{div} \mathcal{L}\left(\Delta_{-1} v \tilde{\Delta}_{-1} \rho\right)-\Delta_{-1} v \cdot \mathcal{L} \nabla \tilde{\Delta}_{-1} \rho
$$


Because the Fourier transform of this quantity is supported in a fixed ball, we obtain by Bernstein's inequality

$$
\begin{aligned}
\left\|\Pi_{3}^{2}\right\|_{L^{\infty}} & \lesssim\left\|\Pi_{3}^{2}\right\|_{L^{2}} \\
& \lesssim\left\|\Delta_{-1} v\right\|_{L^{\infty}}\left\|\tilde{\Delta}_{-1} \rho\right\|_{L^{2}} \\
& \lesssim\|v\|_{L^{2}}\|\rho\|_{L^{2}} .
\end{aligned}
$$

Therefore we get

$$
\left\|\Pi_{3}^{2}\right\|_{C^{\varepsilon}} \lesssim\|v\|_{L^{2}}\|\rho\|_{L^{2}}
$$

Consequently we find

$$
\left\|\Pi_{3}\right\|_{C^{\varepsilon}} \lesssim\|v\|_{L^{2}}\|\rho\|_{L^{2}}+\|\omega\|_{L^{\infty}}\|\rho\|_{L^{p}} .
$$

Combining (3.1), (3.4), and (3.7) yields

$$
\|[\mathcal{L}, v \cdot \nabla] \rho\|_{C^{\varepsilon}} \lesssim\|v\|_{L^{2}}\|\rho\|_{L^{2}}+\|\omega\|_{L^{2} \cap L^{\infty}}\|\rho\|_{L^{p}}
$$

This completes the proof of the commutator estimate.

Now we will prove the following lemma.

LEMma 3.3. Let $f$ and $g$ be two smooth functions. Then, for all $(p, r) \in[2, \infty]^{2}$ such that $p \geq r$ and for $q \in \mathbb{N} \cup\{-1\}$,

$$
\left\|\left[\Delta_{q}, f\right] g\right\|_{L^{p}} \lesssim 2^{q\left(\frac{2}{r}-\frac{2}{p}\right)}\|\nabla f\|_{L^{r}}\|g\|_{L^{2}} .
$$

Proof. According to the definition of the commutator, and using Taylor's formula,

$$
\begin{aligned}
\left\{\left[\Delta_{q}, f\right] g\right\}(x) & =\int_{\mathbb{R}^{2}} \phi_{q}(x-y) g(y)(f(y)-f(x)) d y \\
& =\int_{0}^{1} \int_{\mathbb{R}^{2}} g(y) \Phi_{q}(x-y) \cdot \nabla f(x+t(y-x)) d y d t
\end{aligned}
$$

with

$$
\phi_{q}(x)=2^{2 q} \phi\left(2^{q} x\right), \quad \Phi_{q}(x)=x \phi_{q}(x), \quad \phi \in \mathcal{S}\left(\mathbb{R}^{2}\right) .
$$

Using Hölder's inequality and a change of variables we get, with the notation $\Phi_{q, t}=$ $t^{-2} \Phi_{q}\left(\frac{x}{t}\right)$

$$
\begin{aligned}
\left|\left[\Delta_{q}, f\right] g(x)\right| & \leq \int_{0}^{1} \int_{\mathbb{R}^{2}}\left(|g(y)|\left|\Phi_{q}(x-y)\right|^{\frac{1}{2}}\right)\left(|\nabla f(x+t(y-x))|\left|\Phi_{q}(x-y)\right|^{\frac{1}{2}}\right) d y d t \\
& \left.\leq\left(\int_{\mathbb{R}^{2}}\left|\Phi_{q}(x-y)\right||g(y)|^{2}\right) d y\right)^{\frac{1}{2}} \int_{0}^{1}\left(\int_{\mathbb{R}^{2}}\left|\Phi_{q, t}(y)\right||\nabla f(x-y)|^{2} d y\right)^{\frac{1}{2}} d t \\
& \leq\left(\left|\Phi_{q}\right| \star|g|^{2}\right)^{\frac{1}{2}}(x) \int_{0}^{1}\left(\left|\Phi_{q, t}\right| \star|\nabla f|^{2}\right)^{\frac{1}{2}}(x) d t .
\end{aligned}
$$

It follows from the convolution laws that

$$
\left\|\left[\Delta_{q}, f\right] g\right\|_{L^{p}} \leq\left\|\left|\Phi_{q}\right| \star|g|^{2}\right\|_{L^{\infty}}^{\frac{1}{2}} \int_{0}^{1}\left\|\left(\left|\Phi_{q, t}\right| \star|\nabla f|^{2}\right)^{\frac{1}{2}}\right\|_{L^{p}} d t
$$




$$
\leq\left\|\Phi_{q}\right\|_{L^{\infty}}^{\frac{1}{2}}\|g\|_{L^{2}}\|\nabla f\|_{L^{r}} \int_{0}^{1}\left\|\Phi_{q, t}\right\|_{L^{\rho}}^{\frac{1}{2}} d t
$$

with the assumption $1+\frac{2}{p}=\frac{2}{r}+\frac{1}{\rho}$, which admits a solution despite the fact that $p \geq r$. Straightforward computations yield

$$
\left\|\Phi_{q}\right\|_{L^{\infty}}=2^{q}\|x \phi\|_{L^{\infty}} \quad \text { and } \quad\left\|\Phi_{q, t}\right\|_{L^{\rho}}=t^{-2+\frac{2}{\rho}} 2^{q\left(1-\frac{2}{\rho}\right)}\|x \phi\|_{L^{\rho}} .
$$

Because $\rho<\infty$ one gets

$$
\begin{aligned}
\left\|\left[\Delta_{q}, f\right] g\right\|_{L^{p}} & \leq C\|g\|_{L^{2}}\|\nabla f\|_{L^{r}} 2^{q\left(1-\frac{1}{\rho}\right)} \int_{0}^{1} t^{-1+\frac{1}{\rho}} d t \\
& \leq C\|g\|_{L^{2}}\|\nabla f\|_{L^{r}} 2^{q\left(\frac{2}{r}-\frac{2}{p}\right)} .
\end{aligned}
$$

The following result can be obtained easily from Lemma 3.3.

CoROllary 3.4. Let $v$ be a smooth divergence-free vector field on $\mathbb{R}^{2}$ with vorticity $\omega$ and $f$ be a smooth scalar function. Then, for all $(p, r) \in\left[2, \infty\left[{ }^{2}\right.\right.$ such that $p \geq r$ and for $q \in \mathbb{N} \cup\{-1\}$,

$$
\left\|\left[\Delta_{q}, v\right] f\right\|_{L^{p}} \lesssim 2^{q\left(\frac{2}{r}-\frac{2}{p}\right)}\|\omega\|_{L^{r}}\|f\|_{L^{2}}
$$

\section{Asymptotic behavior}

In this section we intend to establish some time decay estimates for any solution of a transport-diffusion model. We will see afterwards how this allows one to get some global a priori estimates for the vorticity of the system (1.2) which are very close to the ones known for the usual Euler equations.

Proposition 4.1. Let $v$ be a smooth vector field with zero divergence and $\rho$ be a smooth solution of the transport-diffusion equation

$$
\partial_{t} \rho+v \cdot \nabla \rho-\Delta \rho=0, \quad \rho(0, x)=\rho_{0}(x),
$$

such that $\rho_{0} \in L^{1} \cap L^{2}$. Then the following assertions hold true.

(1) For every $t \geq 0$ and $0<\alpha<1$,

$$
(1+t)^{\alpha}\|\rho(t)\|_{L^{2}}^{2}+\int_{0}^{t}(1+\tau)^{\alpha-1}\|\rho(\tau)\|_{L^{2}}^{2} d \tau+\int_{0}^{t}(1+\tau)^{\alpha}\|\rho(\tau)\|_{\dot{H}^{1}}^{2} d \tau \lesssim\left\|\rho_{0}\right\|_{L^{1} \cap L^{2}}^{2} .
$$

(2) For every $t \geq 0$,

$$
(1+t)\|\rho(t)\|_{L^{2}}^{2} \lesssim\left\|\rho_{0}\right\|_{L^{1} \cap L^{2}}^{2}
$$

Proof.

(1) Taking the $L^{2}$ scalar product of the equation (4.1) with $\rho$ and using the incompressibility condition $\operatorname{div} v=0$ we obtain

$$
\frac{d}{d t}\|\rho(t)\|_{L^{2}}^{2}+2\|\nabla \rho(t)\|_{L^{2}}^{2}=0 .
$$


Multiplying this differential equation by $(1+t)^{\alpha}$ and integrating by parts in time yields

$$
(1+t)^{\alpha}\|\rho(t)\|_{L^{2}}^{2}+2 \int_{0}^{t}(1+\tau)^{\alpha}\|\nabla \rho(\tau)\|_{L^{2}}^{2} d \tau=\left\|\rho_{0}\right\|_{L^{2}}^{2}+\alpha \int_{0}^{t}(1+\tau)^{\alpha-1}\|\rho(\tau)\|_{L^{2}}^{2} d \tau .
$$

Now we use Nash's inequality

$$
\|\rho\|_{L^{2}}^{2} \lesssim\|\rho\|_{L^{1}}\|\nabla \rho\|_{L^{2}}
$$

Because $\|\rho(t)\|_{L^{1}} \leq\left\|\rho_{0}\right\|_{L^{1}}$, we get

$$
\|\rho(t)\|_{L^{2}}^{2} \lesssim\left\|\rho_{0}\right\|_{L^{1}}\|\nabla \rho(t)\|_{L^{2}} .
$$

Thus after combining Hölder's inequality with Young's inequality $|a b| \leq \frac{1}{4} a^{2}+b^{2}$ we obtain

$$
\alpha \int_{0}^{t}(1+\tau)^{\alpha-1}\|\rho(\tau)\|_{L^{2}}^{2} d \tau \leq C\left\|\rho_{0}\right\|_{L^{1}}^{2} \int_{0}^{t}(1+\tau)^{\alpha-2} d \tau+\int_{0}^{t}(1+\tau)^{\alpha}\|\nabla \rho(\tau)\|_{L^{2}}^{2} d \tau .
$$

Under the assumption $\alpha<1$ the inequality (4.4) implies

$$
\alpha \int_{0}^{t}(1+\tau)^{\alpha-1}\|\rho(\tau)\|_{L^{2}}^{2} d \tau \leq C\left\|\rho_{0}\right\|_{L^{1}}^{2}+\int_{0}^{t}(1+\tau)^{\alpha}\|\nabla \rho(\tau)\|_{L^{2}}^{2} d \tau
$$

Plugging this estimate into (4.3) gives

$$
(1+t)^{\alpha}\|\rho(t)\|_{L^{2}}^{2}+\int_{0}^{t}(1+\tau)^{\alpha}\|\nabla \rho(\tau)\|_{L^{2}}^{2} d \tau \lesssim\left\|\rho_{0}\right\|_{L^{1} \cap L^{2}}^{2}
$$

As a by-product of (4.4)

$$
\int_{0}^{t}(1+\tau)^{\alpha-1}\|\rho(\tau)\|_{L^{2}}^{2} d \tau \lesssim\left\|\rho_{0}\right\|_{L^{1} \cap L^{2}}^{2} .
$$

This concludes the proof of the first result.

(2) It is obviously seen that the previous proof does not work in the case $\alpha=1$, and what we are only able to obtain is the following estimate:

$$
(1+t)\|\rho(t)\|_{L^{2}}^{2}+\int_{0}^{t}(1+\tau)\|\nabla \rho(\tau)\|_{L^{2}}^{2} d \tau \lesssim\left\|\rho_{0}\right\|_{L^{1} \cap L^{2}}^{2} \log (2+t) .
$$

We will show that the uniform pointwise estimate for the density remains true. For this purpose we combine (4.2) with Nash's inequality and $\|\rho(t)\|_{L^{1}} \leq\left\|\rho_{0}\right\|_{L^{1}}$,

$$
\frac{d}{d t}\|\rho(t)\|_{L^{2}}^{2}+C_{0}\|\rho(t)\|_{L^{2}}^{4} \leq 0, \quad C_{0}=\frac{1}{C\left\|\rho_{0}\right\|_{L^{1}}^{2}} .
$$

Multiplying (4.6) by $(1+t)$, then

$$
\frac{d}{d t}\left[(1+t)\|\rho(t)\|_{L^{2}}^{2}\right]+C_{0}(1+t)\|\rho(t)\|_{L^{2}}^{4} \leq\|\rho(t)\|_{L^{2}}^{2} .
$$


Setting $\theta(t)=(1+t)\|\rho(t)\|_{L^{2}}^{2}$, we obtain the following differential inequality for $\theta$ :

$$
\frac{d}{d t} \theta(t)+C_{0} \frac{\theta^{2}(t)}{(1+t)}-\frac{\theta(t)}{(1+t)} \leq 0 .
$$

This differential inequality is of Bernoulli's type and by an explicit resolution we infer

$$
\theta(t) \leq \frac{1+t}{\frac{1}{\theta(0)}+C_{0} t} .
$$

Hence we find

$$
\begin{aligned}
(1+t)\|\rho(t)\|_{L^{2}}^{2} & \leq \theta(0)+\frac{1}{C_{0}} \\
& \lesssim\left\|\rho_{0}\right\|_{L^{1} \cap L^{2}}^{2}
\end{aligned}
$$

This completes the proof of the proposition.

Now we intend to obtain some global a priori estimates for the solutions of (1.2).

Proposition 4.2. Let $(\rho, \omega)$ be a smooth solution of (1.2) such that $\rho_{0} \in L^{1} \cap L^{p}$ and $\omega_{0} \in L^{2} \cap L^{p}$ with $p \in[2, \infty]$. Then for $t \geq 0$,

$$
\|\omega(t)\|_{L^{p}}+\|\nabla \rho\|_{L_{t}^{1} L^{p}} \leq C_{0} \log ^{2-\frac{2}{p}}(1+t) .
$$

Proof.

First case: $p \in[2, \infty[$. To get the estimate in the particular case $p=2$, we combine (4.5) with Hölder's inequality

$$
\begin{aligned}
\|\nabla \rho\|_{L_{t}^{1} L^{2}} & \leq \log ^{\frac{1}{2}}(1+t)\left(\int_{0}^{t}(1+\tau)\|\nabla \rho(\tau)\|_{L^{2}}^{2} d \tau\right)^{\frac{1}{2}} \\
& \lesssim\left\|\rho_{0}\right\|_{L^{1} \cap L^{2}}^{2} \log (2+t) .
\end{aligned}
$$

The $L^{2}$-estimate of the vorticity can be achieved easily from the equation

$$
\partial_{t} \omega+v \cdot \nabla \omega=\partial_{1} \rho .
$$

It follows that

$$
\|\omega(t)\|_{L^{2}} \leq\left\|\omega_{0}\right\|_{L^{2}}+\int_{0}^{t}\|\nabla \rho(\tau)\|_{L^{2}} d \tau
$$

From (4.9) we get

$$
\|\omega(t)\|_{L^{2}} \leq C_{0} \log (2+t) .
$$

Now, for the general case of $p$ we will use the structure of the vorticity combined with some smoothing effects of the transport-diffusion equation governing the density. We start with localizing in frequency the equation of $\rho$ :

$$
\partial_{t} \Delta_{q} \rho+v \cdot \Delta_{q} \rho-\Delta \Delta_{q} \rho=-\left[\Delta_{q}, v \cdot \nabla\right] \rho .
$$


Multiplying (4.12) by $\Delta_{q} \rho\left|\Delta_{q} \rho\right|^{p-2}$, integrating by parts and using the fact that $\operatorname{div} v=0$, we obtain

$$
\frac{1}{p} \frac{d}{d t}\left\|\Delta_{q} \rho(t)\right\|_{L^{p}}^{p}+p \int_{\mathbb{R}^{2}}\left|\nabla \Delta_{q} \rho\right|^{2}\left|\Delta_{q} \rho\right|^{p-2} d x \leq \int_{\mathbb{R}^{2}}\left|\Delta_{q} \rho\right|^{p-1}\left|\left[\Delta_{q}, v \cdot \nabla\right] \rho\right| d x .
$$

Now recall from $[5,6]$ the following generalized Bernstein inequality:

$$
2^{2 q}\left\|\Delta_{q} \rho\right\|_{L^{p}}^{p} \lesssim \int_{\mathbb{R}^{2}}\left|\nabla \Delta_{q} \rho\right|^{2}\left|\Delta_{q} \rho\right|^{p-2} d x
$$

Thus we find, by using Hölder's inequality,

$$
\frac{d}{d t}\left\|\Delta_{q} \rho(t)\right\|_{L^{p}}^{p}+c 2^{2 q}\left\|\Delta_{q} \rho\right\|_{L^{p}}^{p} \lesssim\left\|\Delta_{q} \rho\right\|_{L^{p}}^{p-1}\left\|\left[\Delta_{q}, v \cdot \nabla\right] \rho\right\|_{L^{p}} .
$$

This gives

$$
\frac{d}{d t}\left\|\Delta_{q} \rho(t)\right\|_{L^{p}}+c 2^{2 q}\left\|\Delta_{q} \rho\right\|_{L^{p}} \lesssim\left\|\left[\Delta_{q}, v \cdot \nabla\right] \rho\right\|_{L^{p}}
$$

Consequently,

$$
\frac{d}{d t}\left(e^{c t 2^{2 q}}\left\|\Delta_{q} \rho(t)\right\|_{L^{p}}\right) \lesssim e^{c t 2^{2 q}}\left\|\left[\Delta_{q}, v \cdot \nabla\right] \rho\right\|_{L^{p}}
$$

Integrating in time, this differential inequality leads to

$$
\left\|\Delta_{q} \rho(t)\right\|_{L^{p}} \lesssim e^{-c t 2^{2 q}}\left\|\Delta_{q} \rho_{0}\right\|_{L^{p}}+\int_{0}^{t} e^{-c(t-\tau) 2^{2 q}}\left\|\left[\Delta_{q}, v \cdot \nabla\right] \rho(\tau)\right\|_{L^{p}} d \tau .
$$

It follows that

$$
2^{q}\left\|\Delta_{q} \rho(t)\right\|_{L_{t}^{1} L^{p}} \lesssim 2^{-q}\left\|\Delta_{q} \rho_{0}\right\|_{L^{p}}+2^{-q} \int_{0}^{t}\left\|\left[\Delta_{q}, v \cdot \nabla\right] \rho(\tau)\right\|_{L^{p}} d \tau .
$$

Applying Corollary 3.4, the inequality (4.13) becomes

$$
2^{q}\left\|\Delta_{q} \rho(t)\right\|_{L_{t}^{1} L^{p}} \lesssim 2^{-q}\left\|\Delta_{q} \rho_{0}\right\|_{L^{p}}+2^{-2 \frac{q}{p}} \int_{0}^{t}\|\omega(\tau)\|_{L^{2}}\|\nabla \rho(\tau)\|_{L^{2}} d \tau .
$$

Now, let $N \in \mathbb{N}$ be a fixed number that will be chosen later. Using the LittlewoodPaley decomposition, the Bernstein Lemma 2.4, and the estimate (4.14),

$$
\begin{aligned}
\|\nabla \rho(t)\|_{L_{t}^{1} L^{p}} & \leq \sum_{-1 \leq q<N}\left\|\nabla \Delta_{q} \rho(t)\right\|_{L_{t}^{1} L^{p}}+\sum_{q \geq N}\left\|\nabla \Delta_{q} \rho(t)\right\|_{L_{t}^{1} L^{p}} \\
& \lesssim \sum_{-1 \leq q<N} 2^{q\left(1-\frac{2}{p}\right)}\left\|\nabla \Delta_{q} \rho(t)\right\|_{L_{t}^{1} L^{2}}+\sum_{q \geq N} 2^{q}\left\|\Delta_{q} \rho(t)\right\|_{L_{t}^{1} L^{p}} \\
\lesssim & 2^{N\left(1-\frac{2}{p}\right)}\|\nabla \rho(t)\|_{L_{t}^{1} L^{2}}+\sum_{q \geq N} 2^{-q}\left\|\Delta_{q} \rho_{0}\right\|_{L^{p}} \\
& \quad+\sum_{q \geq N} 2^{-2 \frac{q}{p}} \int_{0}^{t}\|\omega(\tau)\|_{L^{2}}\|\nabla \rho(\tau)\|_{L^{2}} d \tau \\
\lesssim & \left\|\rho_{0}\right\|_{L^{p}}+2^{N\left(1-\frac{2}{p}\right)}\|\nabla \rho(t)\|_{L_{t}^{1} L^{2}}+2^{-2 \frac{N}{p}} \int_{0}^{t}\|\omega(\tau)\|_{L^{2}}\|\nabla \rho(\tau)\|_{L^{2}} d \tau
\end{aligned}
$$


Then in view of (4.11) we obtain

$$
\|\nabla \rho(t)\|_{L_{t}^{1} L^{p}} \leq\left\|\rho_{0}\right\|_{L^{p}}+\|\nabla \rho(t)\|_{L_{t}^{1} L^{2}}\left(2^{N\left(1-\frac{2}{p}\right)}+2^{-2 \frac{N}{p}} \log (1+t)\right) .
$$

We choose $2^{N} \approx \log (1+t)$, and from (4.9) we obtain

$$
\|\nabla \rho(t)\|_{L_{t}^{1} L^{p}} \leq C_{0} \log ^{2-\frac{2}{p}}(2+t) .
$$

To get the $L^{p}$-estimate for the vorticity we combine (4.15) with

$$
\|\omega(t)\|_{L^{p}} \leq\left\|\omega_{0}\right\|_{L^{p}}+\int_{0}^{t}\|\nabla \rho(\tau)\|_{L^{p}} d \tau .
$$

Therefore,

$$
\|\omega(t)\|_{L^{p}} \leq C_{0} \log ^{2-\frac{2}{p}}(2+t) .
$$

Second case: $p=\infty$. If $r>2$, then by using Bernstein's inequality combined with the smoothing effect (4.13) we get

$$
\begin{aligned}
& \|\nabla \rho(t)\|_{L_{t}^{1} L^{\infty}} \\
\leq & \sum_{-1 \leq q<N}\left\|\nabla \Delta_{q} \rho(t)\right\|_{L_{t}^{1} L^{\infty}}+\sum_{q \geq N}\left\|\nabla \Delta_{q} \rho(t)\right\|_{L_{t}^{1} L^{\infty}} \\
\leq & \sum_{-1 \leq q<N} 2^{2 \frac{q}{r}}\left\|\nabla \Delta_{q} \rho(t)\right\|_{L_{t}^{1} L^{r}}+\sum_{q \geq N} 2^{q\left(\frac{2}{r}+1\right)}\left\|\Delta_{q} \rho(t)\right\|_{L_{t}^{1} L^{r}} \\
\leq & \sum_{-1 \leq q<N} 2^{2 \frac{q}{r}}\left\|\nabla \Delta_{q} \rho(t)\right\|_{L_{t}^{1} L^{r}}+\sum_{q \geq N} 2^{q\left(\frac{2}{r}-1\right)}\left(\left\|\Delta_{q} \rho_{0}\right\|_{L^{r}}+\int_{0}^{t}\left\|\left[\Delta_{q}, v \cdot \nabla\right] \rho(\tau)\right\|_{L^{r}} d \tau\right) .
\end{aligned}
$$

Applying the estimate (4.15), (4.17), and Corollary 3.4 we find

$$
\begin{aligned}
& \|\nabla \rho(t)\|_{L_{t}^{1} L^{\infty}} \\
\leq & \left\|\rho_{0}\right\|_{L^{r}}+\sum_{-1 \leq q<N} 2^{\frac{2 q}{r}}\left\|\nabla \Delta_{q} \rho(t)\right\|_{L_{t}^{1} L^{r}}+\sum_{q \geq N} 2^{q\left(\frac{2}{r}-1\right)} \int_{0}^{t}\|\omega(\tau)\|_{L^{r}}\|\nabla \rho(\tau)\|_{L^{2}} d \tau \\
\leq & \left\|\rho_{0}\right\|_{L^{r}}+2^{2 \frac{N}{r}}\|\nabla \rho\|_{L_{t}^{1} L^{r}}+2^{N\left(\frac{2}{r}-1\right)}\|\omega\|_{L_{t}^{\infty} L^{r}}\|\nabla \rho\|_{L_{t}^{1} L^{2}} \\
\leq & \left\|\rho_{0}\right\|_{L^{r}}+C_{0} 2^{2 \frac{N}{r}} \log ^{2-\frac{2}{r}}(2+t)\left(1+2^{-N} \log (2+t)\right) .
\end{aligned}
$$

Taking $2^{N} \approx \log (2+t)$ we infer that

$$
\|\nabla \rho(t)\|_{L_{t}^{1} L^{\infty}} \lesssim C_{0} \log ^{2}(2+t),
$$

As a direct consequence

$$
\|\omega(t)\|_{L^{\infty}} \lesssim C_{0} \log ^{2}(2+t) .
$$

This completes the proof of the proposition.

Corollary 4.3. Let $\Omega$ be a bounded domain, $\rho_{0} \in L^{1} \cap L^{p}$ with $p>2$, and $\omega_{0}=\mathbf{1}_{\Omega}$. Then any smooth solution of (1.2) satisfies

$$
\left\|\omega(t)-\mathbf{1}_{\Omega_{t}}\right\|_{L^{p}} \leq C_{0} \log ^{2-\frac{2}{p}}(2+t),
$$


where $\Omega_{t}$ is the image of $\Omega$ by the flow $\psi$ associated to the velocity $v$.

Proof. Let $\psi$ be the corresponding flow to the velocity $v$. Then in view of the vorticity equation we have

$$
\omega(t, x)=\omega_{0}\left(\psi^{-1}(t, x)\right)+\int_{0}^{t} \partial_{1} \rho\left(\tau, \psi\left(\tau, \psi^{-1}(t, x)\right)\right) d x .
$$

Because the flow $\psi$ preserves Lebesgue measure we get, from (4.15),

$$
\begin{aligned}
\left\|\omega(t)-\mathbf{1}_{\Omega_{t}}\right\|_{L^{p}} & \leq \int_{0}^{t}\|\nabla \rho(\tau)\|_{L^{p}} d \tau \\
& \leq C_{0} \log ^{2-\frac{2}{p}}(2+t) .
\end{aligned}
$$

\section{Vortex patches}

This section is devoted to the proof of the main result of this paper stated in Theorem 1.2. We will follow the ideas of Chemin developed for the incompressible Euler equations. Before going further into the details of the proof we will recall some basic definitions in the study of the vortex patch problem.

\subsection{General formalism.}

Definition 5.1. Let $\varepsilon \in] 0,1\left[\right.$ and $X=\left(X_{\lambda}\right)_{\lambda \in \Lambda}$ be a family of vector fields such that for every $\lambda \in \Lambda, X_{\lambda}$ and $\operatorname{div} X_{\lambda}$ belong to $C^{\epsilon}$. This family is called admissible if and only if

$$
I(X) \triangleq \inf _{x \in \mathbb{R}^{d}} \sup _{\lambda \in \Lambda}\left|X_{\lambda}(x)\right|>0
$$

We set

$$
\widetilde{\|} X_{\lambda}\left\|_{C^{\varepsilon}}=\right\| X_{\lambda}\left\|_{C^{\varepsilon}}+\right\| \operatorname{div} X_{\lambda} \|_{C^{\varepsilon}} .
$$

For $u \in L^{\infty}$ we define the derivative of $u$ along the vector field $X_{\lambda}$ :

$$
\partial_{X_{\lambda}} u=\operatorname{div}\left(u X_{\lambda}\right)-u \operatorname{div} X_{\lambda} .
$$

We will now introduce the anisotropic Hölder spaces $C^{\varepsilon}(X)$ associated to a family of vector fields $X$.

Definition 5.2. Let $\varepsilon \in] 0,1[$ and $X$ be an admissible family of vector fields. We denote by $C^{\varepsilon}(X)$ the space of bounded functions $u$, such that

$$
\forall \lambda \in \Lambda, \partial_{X_{\lambda}} u \in C^{\varepsilon-1} \quad \text { and } \quad \sup _{\lambda \in \Lambda}\left\|\partial_{X_{\lambda}} u\right\|_{C^{\varepsilon-1}}<+\infty .
$$

This space is equipped with the norm

$$
\|u\|_{C^{\varepsilon}(X)}=\frac{1}{I(X)}\left(\|u\|_{L^{\infty}} \sup _{\lambda \in \Lambda} \widetilde{\|} X_{\lambda}\left\|_{C^{\varepsilon}}+\sup _{\lambda \in \Lambda}\right\| \partial_{X_{\lambda}} u \|_{C^{\varepsilon-1}}\right) .
$$

The following result, proven by Chemin [4], is the main key in the study of the vortex patch problem. It relates the Lipschitz norm of an incompressible vector field $v$ to the co-normal regularity of its vorticity. 
TheOREm 5.3. Let $\varepsilon \in] 0,1[$ and $X$ be a family of vector fields as in Definition 5.1. Let $v$ be a divergence-free vector field such that its vorticity $\omega$ satisfies $\omega \in C^{\varepsilon}(X) \cap L^{2}$. Then there exists a constant $C$ depending only on $\varepsilon$, such that

$$
\|\nabla v\|_{L^{\infty}} \leq C\left(\|\omega\|_{L^{2}}+\|\omega\|_{L^{\infty}} \log \left(e+\frac{\|\omega\|_{C^{\varepsilon}(X)}}{\|\omega\|_{L^{\infty}}}\right)\right) .
$$

Proposition 5.4. Let $v$ be a Lipschitzian vector field and $\psi$ its flow. Let $X_{0}=$ $\left(X_{0, \lambda}\right)_{\lambda \in \Lambda}$ a family of vector fields. We define $X_{t}=\left(X_{t, \lambda}\right)_{\lambda \in \Lambda}$ by

$$
X_{t, \lambda}(x)=\psi_{\star}(t) X_{0, \lambda}=\left(X_{0, \lambda}((x, D)) \psi(t)\right)\left(\psi^{-1}(t, x)\right) .
$$

Then

$$
\left(\partial_{t}+v \cdot \nabla\right) X_{t, \lambda}=\partial_{X_{t, \lambda}} v
$$

Notation: we will sometimes use the notation $X_{\lambda}(t)$ to denote $X_{t, \lambda}$.

The following result describes the commutation between any vector field satisfying (5.2) and the advective operator $v \cdot \nabla$.

Lemma 5.5. Let $(v, \rho)$ be a solution of the system (1.1) and $X_{t} \triangleq\left(X_{t, \lambda}\right)_{\lambda \in \Lambda}$ be a family of vector fields satisfying the equations (5.2). Then we have

$$
\left(\partial_{t}+v \cdot \nabla\right) \partial_{X_{t, \lambda}} \omega=\partial_{X_{t, \lambda}} \partial_{1} \rho .
$$

Proof. By definition we have

$$
\begin{aligned}
\left(\partial_{t}+v \cdot \nabla\right) \partial_{X_{t, \lambda}} \omega & =\left(\partial_{t} X_{t, \lambda}+v \cdot \nabla X_{t, \lambda}\right) \cdot \nabla \omega+X_{t, \lambda} \cdot\left\{\left(\partial_{t}+v \cdot \nabla\right) \nabla \omega\right\} \\
& =\left(\partial_{t} X_{t, \lambda}+v \cdot \nabla X_{t, \lambda}\right) \nabla \omega+X_{t, \lambda} \cdot \nabla\left(\partial_{t} \omega+v \cdot \nabla \omega\right)-\left(X_{t, \lambda} \cdot \nabla v\right) \cdot \nabla \omega .
\end{aligned}
$$

By the virtue of (5.2) and the vorticity equation we obtain

$$
\begin{aligned}
\left(\partial_{t}+v \cdot \nabla\right) \partial_{X_{t, \lambda}} \omega & =\partial_{X_{t, \lambda}} v \cdot \nabla \omega+X_{t, \lambda} \cdot \nabla \partial_{1} \rho-\left(X_{t, \lambda} \cdot \nabla v\right) \cdot \nabla \omega \\
& =X_{t, \lambda} \cdot \nabla \partial_{1} \rho .
\end{aligned}
$$

This completes the proof.

5.2. General statement. We will now give a generalization of the Theorem 1.2 which allows us to deal with more general structures than the vortex patches, for example $\omega=f(x) \mathbf{1}_{\Omega}$ with smooth scalar function $f$.

THEOREM 5.6. Let $0<\varepsilon<1$ and $X_{0}$ be a family of admissible vector fields, $v_{0}$ be a free vector field such that $\omega_{0} \in L^{2} \cap C^{\varepsilon}\left(X_{0}\right)$. Let $\rho_{0} \in L^{1} \cap L^{\infty}$. Then the system (1.1) admits a unique global solution $(v, \rho) \in L_{\text {loc }}^{\infty}\left(\mathbb{R}_{+} ;\right.$Lip $) \times L_{\text {loc }}^{\infty}\left(\mathbb{R}_{+} ; L^{1} \cap L^{\infty}\right)$. More precisely,

$$
\begin{gathered}
\|\nabla v(t)\|_{L^{\infty}} \leq C_{0} e^{C_{0} t \log ^{2}(2+t)} \\
\|\omega(t)\|_{C^{\varepsilon}\left(X_{t}\right)}+\left\|\partial_{X_{0, \lambda}} \psi(t)\right\|_{C^{\varepsilon}} \leq C_{0} e^{\exp \left\{C_{0} t \log ^{2}(2+t)\right\}}
\end{gathered}
$$


Proof. We will restrict ourselves to the proof of the a priori estimates. The existence part of the theorem is classical and can be done for example by using a standard recursive method. We start with using a hidden structure in (1.2) in the spirit of the work [14]. We introduce the coupled function

$$
\Gamma=\omega-\mathcal{L} \rho,
$$

where $\mathcal{L}$ denotes the singular operator $\mathcal{L} \triangleq \partial_{1} \Delta^{-1}$. It is easy to see that $\Gamma$ satisfies

$$
\left(\partial_{t}+v \cdot \nabla\right) \Gamma=[\mathcal{L}, v \cdot \nabla] \rho .
$$

According to the Lemma 5.5, $X_{t}$ commutes with the transport part and consequently

$$
\left(\partial_{t}+v \cdot \nabla\right) \partial_{X_{t, \lambda}} \Gamma=\partial_{X_{t, \lambda}}\{[\mathcal{L}, v \cdot \nabla] \rho\} .
$$

Hence in view of Proposition 2.5 we find

$$
\begin{aligned}
\left\|\partial_{X_{t, \lambda}} \Gamma(t)\right\|_{C^{\varepsilon-1}} \leq & C e^{C V(t)}\left\|\partial_{X_{0, \lambda}} \Gamma_{0}\right\|_{C^{\varepsilon-1}} \\
& +C e^{C V(t)} \int_{0}^{t} e^{-C V(\tau)}\left\|\partial_{X_{t, \lambda}}\{[\mathcal{L}, v \cdot \nabla] \rho(\tau)\}\right\|_{C^{\varepsilon-1}} d \tau
\end{aligned}
$$

with $V(t)=\int_{0}^{t}\|\nabla v(\tau)\|_{L^{\infty}} d \tau$. Now because $C^{\varepsilon}$ is an algebra then we get the general fact

$$
\begin{aligned}
\left\|\partial_{X_{\lambda}} u\right\|_{C^{\varepsilon-1}} & \leq\left\|\operatorname{div}\left(u X_{\lambda}\right)\right\|_{C^{\varepsilon-1}}+\left\|u \operatorname{div} X_{\lambda}\right\|_{C^{\varepsilon-1}} \\
& \lesssim\left\|u X_{\lambda}\right\|_{C^{\varepsilon}}+\left\|u \operatorname{div} X_{\lambda}\right\|_{L^{\infty}} \\
& \lesssim\|u\|_{C^{\varepsilon}} \widetilde{\|} X_{\lambda} \|_{C^{\varepsilon}}
\end{aligned}
$$

Consequently we obtain

$$
\left\|\partial_{X_{t, \lambda}}\{[\mathcal{L}, v \cdot \nabla] \rho\}\right\|_{C^{\varepsilon-1}} \leq C \widetilde{\|} X_{t, \lambda}\left\|_{C^{\varepsilon}}\right\|[\mathcal{L}, v \cdot \nabla] \rho \|_{C^{\varepsilon}} .
$$

The estimate of the commutator term is described in Lemma 3.2:

$$
\|[\mathcal{L}, v \cdot \nabla] \rho\|_{C^{\varepsilon}} \lesssim\|v\|_{L^{2}}\|\rho\|_{L^{2}}+\|\omega\|_{L^{2} \cap L^{\infty}}\|\rho\|_{L^{p}}, \quad p>\frac{2}{1-\varepsilon} .
$$

To estimate the first term of the right-hand side we write according to (4.7)

$$
\begin{aligned}
\|v(t)\|_{L^{2}} & \leq\left\|v_{0}\right\|_{L^{2}}+\int_{0}^{t}\|\rho(\tau)\|_{L^{2}} d \tau \\
& \leq C_{0}(1+t)^{\frac{1}{2}} .
\end{aligned}
$$

Again, by using (4.7),

$$
\|v(t)\|_{L^{2}}\|\rho(t)\|_{L^{2}} \leq C_{0} .
$$

Now applying a usual interpolation inequality combined with the maximum principle and (4.7) we get, for $p \in[2,+\infty[$,

$$
\|\rho(t)\|_{L^{p}} \leq\|\rho(t)\|_{L^{2}}^{\frac{2}{p}}\left\|\rho_{0}\right\|_{L^{\infty}}^{1-\frac{2}{p}} \leq C_{0}(1+t)^{-\frac{1}{p}} .
$$


Thus it follows from the previous estimates, Proposition 4.1-(3), and (5.5) that

$$
\|[\mathcal{L}, v \cdot \nabla] \rho\|_{C^{\varepsilon}} \leq C_{0}+C_{0}(1+t)^{-\frac{1}{p}} \log ^{2}(2+t) \leq C_{0} .
$$

Inserting this estimate into (5.4) yields

$$
\int_{0}^{t} e^{-C V(\tau)}\left\|\partial_{X_{t, \lambda}}\{[\mathcal{L}, v \cdot \nabla] \rho(\tau)\}\right\|_{C^{\varepsilon-1}} d \tau \leq C_{0} \int_{0}^{t} e^{-C V(\tau)}\left\|X_{\lambda}(\tau)\right\|_{C^{\varepsilon}} d \tau .
$$

Substituting (5.6) into (5.3) gives

$$
\left\|\partial_{X_{t, \lambda}} \Gamma(t)\right\|_{C^{\varepsilon-1}} \lesssim e^{C V(t)}\left(\left\|\partial_{X_{0, \lambda}} \Gamma_{0}\right\|_{C^{\varepsilon-1}}+\int_{0}^{t} e^{-C V(\tau)}\left\|X_{\lambda}(\tau)\right\|_{C^{\varepsilon}} d \tau\right) .
$$

By the definition we get

$$
\begin{aligned}
\left\|\partial_{X_{0, \lambda}} \Gamma_{0}\right\|_{C^{\varepsilon-1}} & \leq\left\|\partial_{X_{0, \lambda}} \omega_{0}\right\|_{C^{\varepsilon-1}}+\left\|\partial_{X_{0, \lambda}} \mathcal{L} \rho_{0}\right\|_{C^{\varepsilon-1}} \\
& \leq\left\|\partial_{X_{0, \lambda}} \omega_{0}\right\|_{C^{\varepsilon-1}}+\left\|X_{0, \lambda}\right\|_{C^{\varepsilon}}\left\|\mathcal{L} \rho_{0}\right\|_{C^{\varepsilon}} .
\end{aligned}
$$

Similarly one obtains

$$
\left\|\partial_{X_{t, \lambda}} \mathcal{L} \rho(t)\right\|_{C^{\varepsilon-1}} \leq \widetilde{\|} X_{\lambda}(t)\left\|_{C^{\varepsilon}}\right\| \mathcal{L} \rho(t) \|_{C^{\varepsilon}}
$$

Now we can use the integral representation of the operator $\mathcal{L}$ leading to the pointwise inequality

$$
\left|\mathcal{L} \rho_{0}(x)\right| \lesssim\left(\frac{1}{|\cdot|} \star\left|\rho_{0}\right|\right)(x)
$$

It follows that

$$
\left\|\mathcal{L} \rho_{0}\right\|_{L^{\infty}} \lesssim\left\|\rho_{0}\right\|_{L^{1} \cap L^{\infty}}
$$

Now by the definition of Besov spaces and according to (5.9) and to Bernstein's inequality we get, for $p \geq \frac{2}{1-\varepsilon}$,

$$
\begin{aligned}
\|\mathcal{L} \rho(t)\|_{C^{\varepsilon}} & \leq\|\mathcal{L} \rho(t)\|_{L^{\infty}}+\sup _{q \in \mathbb{N}} 2^{q \varepsilon}\left\|\Delta_{q} \mathcal{L} \rho(t)\right\|_{L^{\infty}} \\
& \lesssim\|\rho(t)\|_{L^{1} \cap L^{\infty}}+\sup _{q \in \mathbb{N}} 2^{q\left(\varepsilon-1+\frac{2}{p}\right)}\left\|\Delta_{q} \rho(t)\right\|_{L^{p}} \\
& \lesssim\left\|\rho_{0}\right\|_{L^{1} \cap L^{\infty}} .
\end{aligned}
$$

This implies

$$
\left\|\partial_{X_{t, \lambda}} \mathcal{L} \rho(t)\right\|_{C^{\varepsilon-1}} \leq C_{0} \widetilde{\|} X_{\lambda}(t) \|_{C^{\varepsilon}} .
$$

Combining (5.7) and (5.8) with (5.10) we find

$$
\begin{aligned}
\left\|\partial_{X_{t, \lambda}} \omega(t)\right\|_{C^{\varepsilon-1}} & \leq\left\|\partial_{X_{t, \lambda}} \Gamma(t)\right\|_{C^{\varepsilon-1}}+\left\|\partial_{X_{t, \lambda}} \mathcal{L} \rho(t)\right\|_{C^{\varepsilon-1}} \\
& \leq C_{0} \widetilde{\|} X_{\lambda}(t) \|_{C^{\varepsilon}}+C_{0} e^{C V(t)}\left(1+\int_{0}^{t} e^{-C V(\tau)} \widetilde{\|} X_{\lambda}(\tau) \|_{C^{\varepsilon}} d \tau\right)
\end{aligned}
$$


It remains to estimate $\widetilde{\|} X_{\lambda}(t) \|_{C^{\varepsilon}}$. Applying Proposition 2.5 to the equation (5.2) we get

$$
\left\|X_{\lambda}(t)\right\|_{C^{\varepsilon}} \leq C e^{C V(t)}\left(\left\|X_{\lambda}(0)\right\|_{C^{\varepsilon}}+\int_{0}^{t} e^{-C V(\tau)}\left\|\partial_{X_{\lambda}} v(\tau)\right\|_{C^{\varepsilon}} d \tau\right) .
$$

Recall from [4] the following inequality:

$$
\left\|\partial_{X_{\lambda}} v\right\|_{C^{\varepsilon}} \leq C\left(\left\|\partial_{X_{\lambda}} \omega\right\|_{C^{\varepsilon-1}}+\left\|\operatorname{div} X_{\lambda}\right\|_{C^{\varepsilon}}\|\omega\|_{L^{\infty}}+\left\|X_{\lambda}\right\|_{C^{\varepsilon}}\|\nabla v\|_{L^{\infty}}\right) .
$$

Inserting (5.13) into (5.12) and using Gronwall's inequality we find

$$
\begin{aligned}
\left\|X_{\lambda}(t)\right\|_{C^{\varepsilon}} \leq C e^{C V(t)}\left\|X_{\lambda}(0)\right\|_{C^{\varepsilon}} & \\
& +C e^{C V(t)} \int_{0}^{t} e^{-C V(\tau)}\left(\left\|\partial_{X_{\lambda}} \omega(\tau)\right\|_{C^{\varepsilon-1}}+\left\|\operatorname{div} X_{\lambda}(\tau)\right\|_{C^{\varepsilon}}\|\omega(\tau)\|_{L^{\infty}}\right) d \tau .
\end{aligned}
$$

To get a bound for $\|\operatorname{div} X(t)\|_{C^{\varepsilon}}$ we apply the divergence operator to (5.2):

$$
\left(\partial_{t}+v \cdot \nabla\right) \operatorname{div} X_{\lambda}(t)=0 .
$$

Thus we obtain, in view of Proposition 2.5,

$$
\left\|\operatorname{div} X_{\lambda}(t)\right\|_{C^{\varepsilon}} \leq C e^{C V(t)}\left\|\operatorname{div} X_{\lambda}(0)\right\|_{C^{\varepsilon}} .
$$

Combining the inequalities (5.14) and (5.15) yields

$$
\begin{aligned}
\widetilde{\|} X_{\lambda}(t) \|_{C^{\varepsilon}} \leq C & e^{C V(t)}\left\|X_{\lambda}(0)\right\|_{C^{\varepsilon}} \\
& +C e^{C V(t)}\left(\left\|\operatorname{div} X_{\lambda}(0)\right\|_{C^{\varepsilon}}\|\omega\|_{L_{t}^{1} L^{\infty}}+\int_{0}^{t} e^{-C V(\tau)}\left\|\partial_{X_{\lambda, \tau}} \omega(\tau)\right\|_{C^{\varepsilon-1}} d \tau\right) .
\end{aligned}
$$

It follows from (4.20) that

$$
\widetilde{\|} X_{\lambda}(t) \|_{C^{\varepsilon}} \leq C_{0} e^{C V(t)}\left((1+t) \log ^{2}(2+t)+\int_{0}^{t} e^{-C V(\tau)}\left\|\partial_{X_{\lambda, \tau}} \omega(\tau)\right\|_{C^{\varepsilon-1}} d \tau\right) .
$$

Hence we deduce, from (5.11),

$$
\left.\widetilde{\|} X_{\lambda}(t) \|_{C^{\varepsilon}} \leq C_{0} e^{C V(t)}\left((1+t) \log ^{2}(2+t)+(1+t) \int_{0}^{t} e^{-C V(\tau)} \widetilde{\|} X_{\lambda}(\tau) \|_{C^{\varepsilon}}\right) d \tau\right)
$$

Therefore Gronwall's inequality yields

$$
\widetilde{\|} X_{\lambda}(t) \|_{C^{\varepsilon}} \leq C_{0} e^{C_{0} t^{2}} e^{C V(t)}
$$

Plugging this estimate into (5.11),

$$
\left\|\partial_{X_{t, \lambda}} \omega(t)\right\|_{C^{\varepsilon-1}} \leq C_{0} e^{C_{0} t^{2}} e^{C V(t)}
$$

This gives

$$
\widetilde{\|} X_{\lambda}(t)\left\|_{C^{\varepsilon}}+\right\| \partial_{X_{t, \lambda}} \omega(t) \|_{C^{\varepsilon-1}} \leq C_{0} e^{C_{0} t^{2}} e^{C V(t)} .
$$


To bound $\omega$ in the anisotropic space $C^{\varepsilon}\left(X_{t}\right)$, it remains to give a lower bound for $I\left(X_{t}\right)$. For this purpose we use the following estimate from [4]:

$$
I\left(X_{t}\right) \geq I\left(X_{0}\right) e^{-C V(t)} .
$$

Consequently we obtain

$$
\|\omega(t)\|_{C^{\varepsilon}\left(X_{t}\right)} \leq C_{0} e^{C_{0} t^{2}} e^{C V(t)}
$$

Using the logarithmic estimate (5.1), (5.17), (4.17), and (4.20), one finds

$$
\begin{aligned}
\|\nabla v(t)\|_{L^{\infty}} & \leq C_{0}\left(\log (2+t)+\log ^{2}(2+t) \log \left(e+\|\omega(t)\|_{C^{\varepsilon}\left(X_{t}\right)}\right)\right) \\
& \leq C_{0}\left(1+t^{2} \log ^{2}(2+t)+\log ^{2}(2+t) \int_{0}^{t}\|\nabla v(\tau)\|_{L^{\infty}} d \tau\right) .
\end{aligned}
$$

Applying Gronwall's lemma leads to

$$
\|\nabla v(t)\|_{L^{\infty}} \leq C_{0} e^{C_{0} t \log ^{2}(2+t)} .
$$

Therefore combining this estimate with (5.17) we get

$$
\|\omega(t)\|_{C^{\varepsilon}\left(X_{t}\right)} \leq C_{0} e^{\exp \left\{C_{0} t \log ^{2}(2+t)\right\}} .
$$

Now it remains to estimate $\partial_{X_{t, \lambda}} \psi(t)$. For this goal we use the identity $\partial_{X_{0, \lambda}} \psi(t)=$ $X_{t, \lambda} \circ \psi(t)$, and thus

$$
\left\|\partial_{X_{0, \lambda}} \psi(t)\right\|_{C^{\varepsilon}}=\left\|X_{t, \lambda} \circ \psi(t)\right\|_{C^{\varepsilon}} .
$$

Using the definition $\|f\|_{C^{\varepsilon}}=\|f\|_{L^{\infty}}+\sup _{x \neq y} \frac{|f(x)-f(y)|}{|x-y|^{\varepsilon}}$, we get

$$
\begin{aligned}
\left\|X_{t, \lambda} \circ \psi(t)\right\|_{C^{\varepsilon}} & \leq\left\|X_{t, \lambda}\right\|_{C^{\varepsilon}}\|\nabla \psi(t)\|_{L^{\infty}}^{\varepsilon} \\
& \leq\left\|X_{t, \lambda}\right\|_{C^{\varepsilon}} e^{C V(t)} .
\end{aligned}
$$

From (5.18) we infer that

$$
\left\|X_{t, \lambda} \circ \psi(t)\right\|_{C^{\varepsilon}} \leq C_{0} e^{\exp \left\{C_{0} t \log ^{2}(2+t)\right\}} .
$$

This concludes the proof of Theorem 5.6.

5.3. Proof of the main result. We shall give the proof of Theorem 1.2. The Lipschitz estimate for the velocity follows immediately from Theorem 5.6. Thus it remains to prove two points. The first one concerns the image by the flow of the initial boundary $\Omega_{t}=\psi(t, \Omega)$ which will preserve its initial regularity through the time evolution. The second one is the estimate of the difference between the vorticity and the patch $\mathbf{1}_{\psi(t, \Omega)}$.

We will start with constructing an admissible family of vector fields $X_{0}$ for which $\omega_{0}=\mathbf{1}_{\Omega} \in C^{\varepsilon}\left(X_{0}\right)$. According to the definition of a $C^{1+\varepsilon}\left(\mathbb{R}^{2}\right)$-bounded domain we claim the existence of a function $f_{0} \in C^{1+\varepsilon}\left(\mathbb{R}^{2}\right)$ such that in a neighborhood $V$ of $\partial \Omega$ we have $\partial \Omega=f_{0}^{-1}(\{0\}) \cap V$ and $\nabla f_{0}(x) \neq 0$ in $V$. Let $\chi$ be a smooth function supported in $V$ and taking the value 1 in a small neighborhood of $\partial \Omega$. We set

$$
X_{0,0}=\nabla^{\perp} f_{0} \quad \text { and } \quad X_{0,1}=(1-\chi)\left(\begin{array}{l}
1 \\
0
\end{array}\right) .
$$


We can easily check that the family $\left\{X_{0,0}, X_{0,1}\right\}$ is admissible in the sense of the Definition 5.1. As the vector field $X_{0,0}$ is Hamiltonian and tangential to the closed curve $\partial \Omega$ then necessarily it will be degenerate at some points inside the domain $\Omega$. For this reason we should complete with the vector field $X_{0,1}$ in order to get a non degenerate family. Now we claim that $\partial_{X_{0, i}} \omega_{0}=0$ for $i \in\{0,1\}$. This is obvious for the vector field $X_{0,1}$ and we shall prove it for $X_{0,0}$ in a weak sense. If $\varphi \in \mathcal{D}\left(\mathbb{R}^{2}\right)$, then according to Green's formula we have

$$
\begin{aligned}
\int_{\mathbb{R}^{2}} \partial_{X_{0,0}} \omega(x) \varphi(x) d x & =\int_{\mathbb{R}^{2}} \operatorname{div}\left(\nabla^{\perp} f_{0} \mathbf{1}_{\Omega}\right) \varphi(x) d x \\
& =-\int_{\Omega} \nabla^{\perp} f_{0} \cdot \nabla \varphi(x) d x \\
& =-\int_{\partial \Omega} \nabla^{\perp} f_{0}(\sigma) \cdot \vec{n}(\sigma) \varphi(\sigma) d \sigma \\
& =0 .
\end{aligned}
$$

Now we will work with a judicious parameterization of $\partial \Omega$. Let $x_{0} \in \partial \Omega$ and define the curve $\gamma^{0}$ by the autonomous differential equation

$$
\left\{\begin{array}{l}
\partial_{\sigma} \gamma^{0}(\sigma)=\nabla^{\perp} f_{0}\left(\gamma^{0}(\sigma)\right) \\
\gamma^{0}(0)=x_{0}
\end{array}\right.
$$

By classical arguments we can see that $\gamma^{0}$ belongs to $C^{1+\varepsilon}\left(\mathbb{R}, \mathbb{R}^{2}\right)$. Let $\gamma(t, \sigma) \triangleq$ $\psi\left(t, \gamma_{0}(\sigma)\right)$, which gives a parameterization of the boundary of $\psi(t, \Omega)$. By differentiation we get

$$
\partial_{\sigma} \gamma(t, \sigma)=\left(\partial_{X_{0,0}} \psi\right)\left(t, \gamma^{0}(\sigma)\right)
$$

From Theorem 5.6 we infer $\partial_{X_{0,0}} \psi \in L_{l o c}^{\infty}\left(\mathbb{R}_{+} ; C^{\varepsilon}\right)$, and thus $\gamma(t)$ belongs to $L_{\text {loc }}^{\infty}\left(\mathbb{R}_{+} ; C^{1+\varepsilon}\right)$. This completes the proof of the regularity persistence of the boundary of $\Omega_{t}$. Let us now move to the proof of the last point dealing with the decomposition of the vorticity into two parts: the vortex patch and a smooth term related to the density. According to the proof of the Corollary 4.3 we have

$$
\begin{aligned}
\omega(t, x) & =\omega_{0}\left(\psi^{-1}(t, x)\right)+\int_{0}^{t}\left(\partial_{1} \rho\right)\left(\tau, \psi\left(\tau, \psi^{-1}(t, x)\right)\right) d \tau \\
& =\omega_{0}\left(\psi^{-1}(t, x)\right)+\int_{0}^{t}\left(\partial_{1} \rho\right)\left(\tau, \psi_{t}(\tau, x)\right) d \tau \\
& =\mathbf{1}_{\psi(t, \Omega)}+\widetilde{\rho}(t, x),
\end{aligned}
$$

with $\widetilde{\rho}(t, x)=\int_{0}^{t}\left(\partial_{1} \rho\right)\left(\tau, \psi_{t}(\tau, x)\right) d \tau$. Here $\psi_{t}(\tau, \cdot)$ is the generalized flow defined by the equation

$$
\left\{\begin{array}{l}
\partial_{\tau} \psi_{t}(\tau, x)=v\left(\tau, \psi_{t}(\tau, x)\right) \\
\psi_{t}(t, x)=x
\end{array}\right.
$$

Recall the classical identities

$$
\psi(\tau, x)=\psi_{0}(\tau, x), \quad \psi^{-1}(\tau, x)=\psi_{\tau}(0, x) \quad \text { and } \quad \psi\left(\tau, \psi^{-1}(t, x)\right)=\psi_{t}(\tau, x) .
$$


Using the generalized flow equation and Gronwall's inequality we get

$$
\left\|\nabla \psi_{t}(\tau)\right\|_{L^{\infty}} \leq \exp \left(\left|\int_{t}^{\tau}\|\nabla v(s)\|_{L^{\infty}} d s\right|\right) .
$$

Now we intend to prove the following estimate: for every $\eta \in[0,1[$, we have

$$
\|\widetilde{\rho}(t)\|_{C^{\eta}} \leq C\|\rho\|_{\widetilde{L}_{t}^{1} B_{\infty, \infty}^{2}} e^{C V(t)}, \quad \text { with } \quad V(t)=\int_{0}^{t}\|\nabla v(\tau)\|_{L^{\infty}} d \tau .
$$

Indeed, the classical composition law

$$
\|f \circ \psi\|_{C^{\eta}} \leq C\|f\|_{C^{\eta}}\left(1+\|\nabla \psi\|_{L^{\infty}}^{\eta}\right),
$$

combined with (5.19), yields

$$
\left\|\left(\partial_{1} \rho\right)\left(\tau, \psi_{t}(\tau, x)\right)\right\|_{C^{\eta}} \leq C\|\rho(\tau)\|_{C^{1+\eta}} \exp \left(C\left|\int_{\tau}^{t}\|\nabla v(s)\|_{L^{\infty}} d s\right|\right) .
$$

It follows that

$$
\|\widetilde{\rho}(t)\|_{C^{\eta}} \leq C\|\rho\|_{L_{t}^{1} C^{1+\eta}} e^{C V(t)} .
$$

From the embedding $\widetilde{L_{t}^{1}} B_{\infty, \infty}^{2} \hookrightarrow L_{t}^{1} C^{1+\eta}$ we obtain

$$
\|\widetilde{\rho}(t)\|_{C^{\eta}} \leq C\|\rho\|_{\widetilde{L_{t}^{1}} B_{\infty}^{2}, \infty} e^{C V(t)} .
$$

Then the estimate (5.18) leads to

$$
\|\widetilde{\rho}(t)\|_{C^{\eta}} \leq C_{0} e^{\exp C_{0} t \log ^{2}(2+t)}\|\rho\|_{\widetilde{L}_{t}^{1} B_{\infty}^{2}, \infty} .
$$

By Proposition 3.1 of [12] we obtain

$$
\begin{aligned}
\|\rho\|_{\widetilde{L_{t}^{1}} B_{\infty, \infty}^{2}} & \leq C\left\|\rho_{0}\right\|_{L^{p}}(1+t+V(t)) \\
& \leq C_{0} e^{C_{0} t \log ^{2}(2+t)} .
\end{aligned}
$$

Consequently

$$
\|\widetilde{\rho}(t)\|_{C^{\eta}} \leq C_{0} e^{\exp C_{0} t \log ^{2}(2+t)} .
$$

This concludes the proof of Theorem 1.2.

\section{REFERENCES}

[1] A.L. Bertozzi and P. Constantin, Global regularity for vortex patches, Commun. Math. Phys., 152(1), 19-28, 1993.

[2] J.M. Bony, Calcul symbolique et propagation des singularités pour les équations aux dérivées partielles non linéaires, Ann. de l'Ecole Norm. Sup., 14, 209-246, 1981.

[3] D. Chae, Global regularity for the 2D-Boussinesq equations with partial viscous terms, Adv. Math., 203(2), 497-513, 2006.

[4] J.Y. Chemin, Perfect Incompressible Fluids, Oxford University Press, 1998.

[5] Q. Chen, C. Miao, and Z. Zhang, A new Bernstein inequality and the 2D dissipative quasigeostrophic equation, Commun. Math. Phys., 271, 821-838, 2007. 
[6] R. Danchin, Poches de tourbillon visqueuses, J. Math. Pures Appl., (9)76, 7, 609-647, 1997.

[7] R. Danchin and M. Paicu, Global well-posedness issues for the inviscid Boussinesq system with Yudovich's type data, Commun. Math. Phys., 290(1), 1-14, 2009.

[8] R. Danchin and M. Paicu, Le théorème de Leary et le théorème de Fujita-Kato pour le système de Boussinesq partiellement visqueux, Bulletin de la S. M. F., 136, 261-309, 2008.

[9] N. Depauw, Poche de tourbillon pour Euler $2 D$ dans un ouvert à bord, J. Math. Pures Appl., (9)78, 3, 313-351, 1999.

[10] P. Gamblin and X. Saint Raymond, On three-dimensional vortex patches, Bull. Soc. Math. France, 123(3), 375-424, 1995

[11] T. Hmidi, Régularité höldérienne des poches de tourbillon visqueuses, J. Math. Pures Appl., (9)84, 11, 1455-1495, 2005.

[12] T. Hmidi and S. Keraani, Inviscid limit for the two-dimensional Navier-Stokes equation in a critical Besov space, Asymp. Anal., 53(3), 125-138, 2007.

[13] T. Hmidi and S. Keraani, On the global well-posedness of the two-dimensional Boussinesq system with a zero diffusivity, Adv. Diff. Eqs., 12(4), 461-480, 2007.

[14] T. Hmidi, S. Keraani, and F. Rousset, Global well-posedness for an Euler-Boussinesq system with critical dissipation, Commun. Part. Diff. Eqs., 36(3), 420-445, 2011.

[15] T. Hmidi, S. Keraani, and F. Rousset, Global well-posedness for a Navier-Stokes-Boussinesq system with critical dissipation, J. Diff. Eqs., 249, 2147-2174, 2010.

[16] T. Hmidi and F. Rousset, Global well-posedness for the Euler-Boussinesq system with axisymmetric data, J. Funct. Anal., 260(3), 745-796, 2011.

[17] T. Hmidi and M. Zerguine, On the global well-posedness of the Euler-Boussinesq system with fractional dissipation, Physica D., 239, 1387-1401, 2010.

[18] A. Larios, E. Lunasin, and E.S. Titi, Global well-posedness for The 2D Boussinesq system without heat diffusion and with either anisotropic viscosity or inviscid Voigt- $\alpha$ regularization, arXiv:1010.5024v1.

[19] C. Miao and L. Xue, On the global well-posedness of a class of Boussinesq- Navier-Stokes systems, NoDEA Nonlinear Diff. Eqs. Appl., 18(6), 707-735, 2011. 